\title{
Entropy, Vortex Interactions and the Phase Diagram of Heavy-Ion Irradiated $\mathrm{Bi}_{2} \mathrm{Sr}_{2} \mathrm{CaCu}_{2} \mathrm{O}_{8+\delta}$
}

\author{
C.J. van der Beek ${ }^{1}$, M. Konczykowski ${ }^{1}$, R.J. Drost ${ }^{2}$, P.H. Kes ${ }^{2}$, \\ A.V. Samoilov ${ }^{3}$, N.Chikumoto ${ }^{4}$, S. Bouffard ${ }^{5}$, M.V. Feigel'man ${ }^{6}$ \\ ${ }^{1}$ Laboratoire des Solides Irradiés, Ecole Polytechnique, 91128 Palaiseau, France \\ ${ }^{2}$ Kamerlingh Onnes Laboratorium, P.O. Box 9506, 2300 RA Leiden, the Netherlands \\ ${ }^{3}$ Condensed Matter Physics 114-36, California Institute of Technology, Pasadena CA 91125, U.S.A. \\ ${ }^{4}$ Superconductivity Research Laboratory, ISTEC, Minato-ku, Tokyo 105, Japan \\ ${ }^{5}$ Centre Interdisciplinaire de Recherche avec les Ions Lourds (C.I.R.I.L.), B.P. 5133, 14040 Caen Cedex, France \\ ${ }^{6}$ Landau Institute of Theoretical Physics, Moscow, Russia
}

\begin{abstract}
Using dynamic and thermodynamic magnetization measurements, we analyze the phase diagram of $\mathrm{Bi}_{2} \mathrm{Sr}_{2} \mathrm{CaCu}_{2} \mathrm{O}_{8+\delta}$ single crystals containing amorphous columnar defects created by heavy-ion irradiation. Reversible magnetization experiments yield the respective magnitudes of the pinning energy and entropy contributions to the free energy of the vortex lattice. It appears that the entropy contribution in the London regime is relatively minor in both unirradiated and irradiated crystals, except in the case of high density of columns and inductions $B$ that are smaller than the interaction field $H_{\text {int }} \approx B_{\phi} / 6$. The dependence of the entropy contribution on vortex- and defect density correlates well with measurements of the irreversibility line $H_{i r r}(T)$, which shows a sharp increase at $H_{i n t}$.
\end{abstract}

\section{Introduction}

The enormous enhancement of the critical current density [1-4] and of the irreversibility line (IRL) separating the vortex glass and liquid phases $[3,4]$ obtained after the introduction of amorphous columnar defects into the layered high-Tc superconductor $\mathrm{Bi}_{2} \mathrm{Sr}_{2} \mathrm{CaCu}_{2} \mathrm{O}_{8+\delta}$ has spurred numerous investigations. Whereas initial studies conducted at temperatures below $20 \mathrm{~K}$ seemed to indicate that flux pinning and dynamics in this sytem could be described within a simple model describing the interaction between single pancake vortices (i.e. the intersections of the vortex lines with the superconducting $\mathrm{CuO}_{2}$-double layers) and the insulating defects $[1,2]$, later work showed that collective effects should be taken into account in order to understand the vortex physics at higher temperatures (above $40 \mathrm{~K}$ ). The field dependence of the sustainable current density [5] can only be explained if the in-plane repulsion between pancakes is taken into account. Further experiments on the dependence of the screening current $\mathrm{j}$ on the angle between the applied magnetic field and the linear tracks demonstrated that above $40 \mathrm{~K}$ pancakes belonging to the same vortex line ("stack") move in a correlated way [6,7]; the stretched-exponential time dependence of the screening current, $j \sim(\ln t)^{-1 / \mu}[4,8]$, supports this finding. Measurements of the c-axis resistivity [9-11] and c-axis critical current, using Josephson Plasma Resonance (JPR) [12-14], show a drastic enhancement of these quantities 
when the field is increased above $B_{\phi} / 6$; this has been interpreted in terms of pancake vortex alignment and an ensuing "recoupling transition" in the vortex liquid phase. It was proposed that the recoupling is driven by the competition between the attractive interaction between pancakes in different layers and the entropy gain obtained by spreading pancakes belonging to the same stack over different columns [14-16]. It must be stressed however that the fields under study are (much) larger than the crossover field $B_{c r} \equiv \Phi_{0} / \gamma^{2} s^{2}$ (with $\gamma \sim 300$ the anisotropy parameter, $s=15 \mathrm{~nm}$ the spacing between $\mathrm{CuO} 2$ double layers in the $\mathrm{Bi}_{2} \mathrm{Sr}_{2} \mathrm{CaCu}_{2} \mathrm{O}_{8+\delta}$ material, and $\Phi_{0}=h / 2 e$ the flux quantum). This means that the interlayer pancake interaction is much weaker than the intralayer interaction. A number of authors have stressed the importance of the intraplane pancake repulsion in determining the vortex configuration and vortex dynamics in the presence of columnar defects $[17,18]$. In this paper, we analyze the reversible magnetization of heavy-ion irradiated $\mathrm{Bi}_{2} \mathrm{Sr}_{2} \mathrm{CaCu}_{2} \mathrm{O}_{8+\delta}$ single crystals. We determine the relative magnitudes of the free energy gain associated with vortex pinning on the irradiation-induced amorphous columnar tracks and the entropy gain associated with the possibility of pancake vortices to occupy different tracks. We show how information on vortex alignment can be obtained from the reversible magnetization, and how the vortex arrangement affects their dynamics and the phase diagram.

\section{Experimental details}

Different batches of $\mathrm{Bi}_{2} \mathrm{Sr}_{2} \mathrm{CaCu}_{2} \mathrm{O}_{8+\delta}$ single crystals were grown using the travelling-solvent floating-zone technique at the University of Amsterdam and the University of Tokyo. A number of crystals were annealed in air at $800 \mathrm{C}$ in order to obtain the optimum $T_{c}=90 \mathrm{~K}$. Other crystals were left as-grown (slightly overdoped) with a $T_{c}$ of $83 \mathrm{~K}$. After imaging of the flux penetration by magneto-optics and the removal of defective regions, a number of large pieces of size $2 \times 1 \mathrm{~mm}^{2} \times 20 \mathrm{~mm}$ were cut for the reversible magnetization experiments. Smaller pieces of size $800 \times 800 \times 20 \mu \mathrm{m}^{3}$ were prepared for AC transmittivity measurements of the irreversibility line (IRL) [8]. The crystals were subsequently irradiated with $5.8 \mathrm{GeV} \mathrm{Pb}^{56+}$ ions at the Grand Accélérateur National d'Ions Lourds (GANIL) in Caen. The ion beam was directed along the $c$-axis; each ion impact created an amorphous columnar track of radius $c_{0} \approx 3.5$ $\mathrm{nm}$, traversing the entire crystal thickness. The ion dose

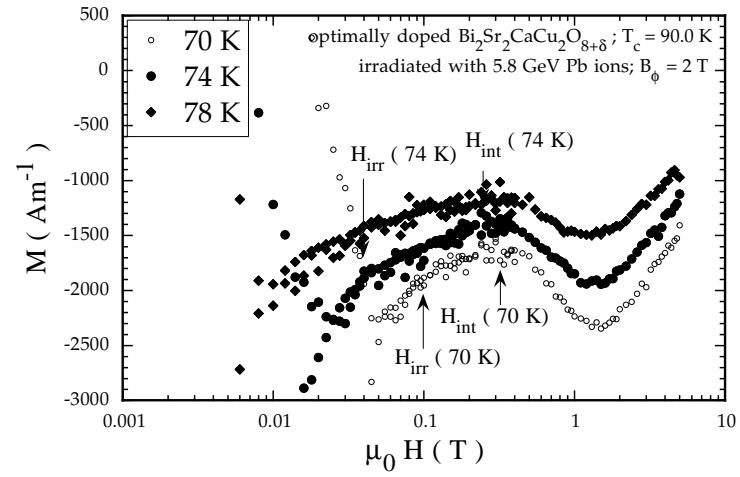

Figure 1: Magnetization of an optimally doped $\mathrm{Bi}_{2} \mathrm{Sr}_{2} \mathrm{CaCu}_{2} \mathrm{O}_{8+\delta}$ single crystal grown at the University of Amsterdam, and irradiated with $5.8 \mathrm{GeV} \mathrm{Pb}$ ions so as to produce a matching field $B_{\phi}=2 \mathrm{~T}$. The arrows denote the irreversibility field $H_{i r r}$ as determined from the high temperature onset of a third harmonic response in field-cooled AC transmittivity experiments, as well as the interaction field $H_{\text {int }}$ above which intervortex repulsion starts to limit the free energy gain that can be obtained from vortex localization on the columnar defects.

for different crystals varied between $1 \times 10^{9}$ and $2 \times 10^{11}$ $\mathrm{cm}^{-2}$, corresponding to dose-equivalent matching fields $B_{\phi} \equiv \Phi_{0} n_{d}$ between $20 \mathrm{mT}$ and $2 \mathrm{~T}\left(n_{d}\right.$ is the columnar defect density). The reversible magnetization was measured in Leiden using a commercial SQUID magnetometer. The IRL's presented below

were determined by ac Local Hall Probe Magnetometry $[8,19]$ using an ac field of amplitude 6 Oe and frequency $7.753 \mathrm{~Hz}$, applied colinearly with dc field and the crystalline $c$-axis. The irreversibility temperature $T_{i r r}(B)$ (or irreversibility field $B_{i r r}(T)$ ) is defined as that at which one first observes a third harmonic in the ac screening current when cooling.

\section{Results and Discussion}

\subsection{Reversible magnetization}

Figure 1 shows the magnetization of an optimally doped $\mathrm{Bi}_{2} \mathrm{Sr}_{2} \mathrm{CaCu}_{2} \mathrm{O}_{8+\delta}$ single crystal with $B_{\phi}=2 \mathrm{~T}$. The reversible magnetization Mrev exhibits the usual features found after heavy-ion irradiation [20,21]. Note that the reversible magnetization corresponds to the vortex chemical potential $\mu$, i.e. $M_{r e v}=-\partial G / \partial B=$ 
$-\Phi_{0}-1 \partial G / \partial n_{v}=-\Phi_{0}^{-1} \mu$ and is therefore equal to the free energy $\Delta G$ needed to insert (or to extract) a vortex into (from) the vortex lattice. At inductions much less than $B_{\phi}$ all vortices can lower their free energy by becoming localized on a defect site, hence the absolute value of the magnetization $\left|M_{r e v}\right|$ is reduced with respect to that of the usual magnetization in the London model, $M_{r e v}^{0}=-\varepsilon_{0} / 2 \Phi_{0} \ln \left(\eta B_{c 2} / e B\right)$, by a corresponding amount $\left(\varepsilon_{0}(T)=\Phi_{0} 2 / 4 \pi \mu_{0} \lambda^{2}(T)\right.$ is the vortex line energy, $\lambda(T)$ is the penetration depth, and $\eta \approx 1$ ). The fact that this amount is large, i.e. of the same order of magnitude as the total magnetization, means that vortices are strongly bound to the columnar defects. As the matching field is approached, favourable sites become rare and more and more "interstitial" vortices (i.e. not localized on a columnar defect) with higher free energy appear. Hence $\left|M_{r e v}\right|$ increases (at the interaction field, labelled $H_{\text {int }}$ in Fig.1). At fields far above $B_{\phi}$, no defect sites are available anymore, all vortices that enter the sample occupy sites in the intercolumn space; hence, the magnetization is comparable to $M_{r e v}^{0}$. The rounding of the magnetization near $B_{\phi}$ is the result of intervortex repulsion, which prohibits the occupation of close-lying columnar defect sites even at fields well below $B_{\phi}[18,20]$, and forces vortices to occupy interstitial sites.

The free energy gain manifest at low fields was initially interpreted as being the sole result of pancake vortex pinning on the columnar tracks. Then, $M_{\text {rev }}(B<<$ $\left.B_{\phi}\right)=M_{r e v}^{0}+U_{0} / \Phi_{0}$, and the pinning energy per unit length $U_{0}$ can be directly obtained as the difference between $M_{\text {rev }}$ (at low field) and the extrapolation to low fields of the magnetization at fields much above $B_{\phi}$. The application of this procedure to irradiated crystals with relatively low $B_{\phi}$ has been used to establish that the main pinning mechanism in heavy-ion irradiated $\mathrm{Bi}_{2} \mathrm{Sr}_{2} \mathrm{CaCu}_{2} \mathrm{O}_{8+\delta}$ arises from the interaction of the vortex core with the defects [22]. Applying the same method to the magnetization of crystals with higher $B_{\phi}$ (as in Fig.1) yields pinning energies that exceed any reasonable theoretical prediction. Conversely, in numerical simulations of column occupation [18] one has to assume an artificially short-ranged vortex interaction in order to arrive at a pronounced magnetization feature such as that in Fig.1. Furthermore, the fact that the logarithmic field derivative of the magnetization in the low field limit is substantially smaller than that at fields larger than $B_{\phi}$ indicates that there must be a second free energy contribution (i.e. entropy) that favours vortex creation and column occupation.

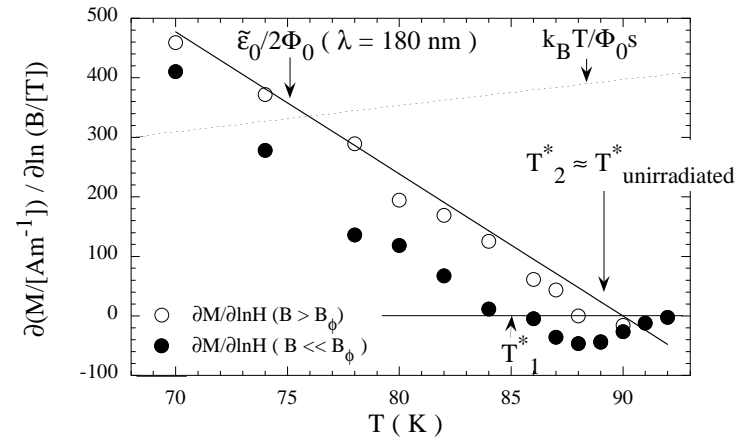

Figure 2: Temperature dependence of the logarithmic field derivative of the reversible magnetization for the same crystal as in Figure 1, in the field regimes $B \ll B_{\phi}$ and $B \gg B_{\phi}$ respectively. The drawn lines represent the value of $\partial M_{r e v}^{0} / \partial \ln B=\varepsilon_{0}(0) / 2 \Phi_{0}$ as extracted from experiment, and the hypothetical entropy contribution $\partial M_{t h} / \partial T \partial \ln B=k_{B} T / \Phi_{0}[23,24]$.

\subsection{Entropy contribution to the magnetization}

The possible entropy contribution Mth to the magnetization of layered superconductors in the London regime, arising from vortex positional fluctuations, was considered by Bulaevskii et al. [23]. They derived that for large fields $B \gg B_{c r}, M_{t h}=\left(k_{B} T / \Phi_{0} s\right) \ln \left(B_{0} / B\right)$, with $B_{0} \approx B_{c 2}\left(\varepsilon_{0} s / k_{B} T\right)$ a characteristic field related to the elemental phase area. As a consequence, the logarithmic field derivative $\partial M_{r e v}^{0} / \partial \ln B$ is cancelled by $\partial M_{t h} / \partial \ln B$ at the "crossing temperature" $T^{*}=$ $T_{c} /\left(1+2 k_{B} T / \varepsilon_{0} s\right) ;$ at $T^{*}$, the reversible magnetization is field-independent. The theory was extended [24] to describe heavy-ion irradiated layered superconductors, which exhibit two distinct crossing temperatures $T_{1}^{*}$ and $T_{2}^{*}[16,21]$, in the field regimes $B<<B_{\phi}$ and $B>>B_{\phi}$ respectively (see Fig.2).

The magnitude of the entropy contribution to the magnetization can be unambiguously evaluated from the temperature derivative of $\partial M_{r e v} / \partial \ln B$. Namely, this is expected to consist of the two contributions $\partial^{2} M_{\text {rev }}^{0} / \partial T \partial \ln B=$ $-\varepsilon_{0}(0) / 2 \Phi_{0} T_{c}$ and $\partial^{2} M_{t h} / \partial T \partial \ln B=k_{B} / \Phi_{0} s$ [25]. The first term can be read from Fig.2, $\partial^{2} M_{r e v}^{0} / \partial T \partial \ln B \approx 23$, and yields the value of the penetration depth at $T=0, \lambda(0)=180 \mathrm{~nm}[26]$. The second contribution is constant and equal to 4.4 $\mathrm{Am}^{-1} \mathrm{~K}^{-1}$; the ratio $4.4 / 23=0.19$ represents the error in $\lambda(0)$ due to the presence of fluctuations. Having 


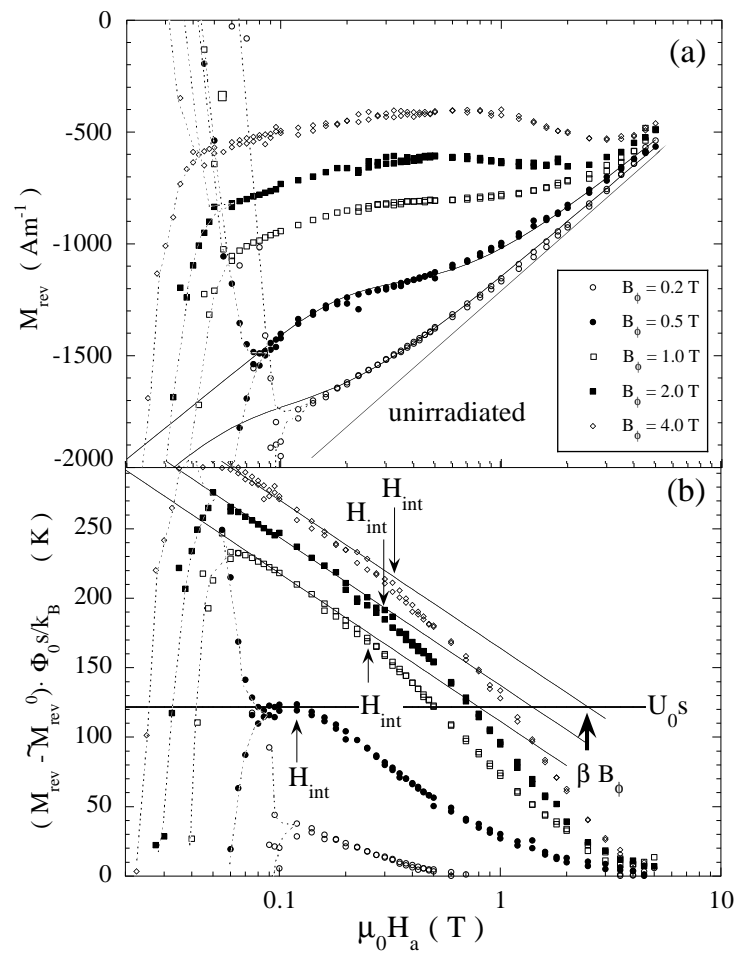

Figure 3: (a) Reversible magnetization of a series of lightly overdoped single crystals (University of Amsterdam, $T_{c}=83$ $\mathrm{K}$ ), irradiated to varying matching fields, as indicated. The drawn lines indicate the magnetization of the unirradiated sample, and fits to the data for $B_{\phi}=0.2 \mathrm{~T}$ and $0.5 \mathrm{~T}$ to Eq. (1) (b) The difference between the experimental magnetization and that of an unirradiated crystal, multiplied by $\Phi_{0} s$. In the low-field limit, this quantity is equal to the sum of the pinning energy and the entropy per pancake. The drawn lines indicate the "bare" pinning energy per pancake U0s (line through data for $B_{\phi}=0.5 \mathrm{~T}$ ), and, for higher $B_{\phi}$, the sum of $U_{0} s$ and the entropy $T S=\left(k_{B} T / \Phi_{0} s\right) \ln \left(\beta B_{\phi} / B\right)$ associated with the fact that individual pancakes belonging to the same vortex line can take advantage of several columnar defect sites. The interaction field $H_{\text {int }}$ is identified as that at which, as a result of intervortex repulsion, pancake vortices belonging to the same stack start to line up on the same columnar defect.

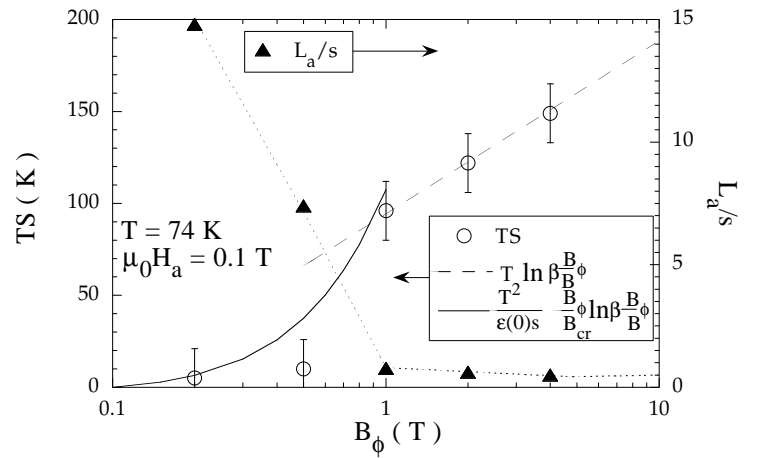

Figure 4: Matching field dependence of the low-field entropy contribution to the free energy (measured at an applied field $\mu_{0} H_{a}=0.1 \mathrm{~T}$ ). The drawn lines indicate the model dependence in the regime where pancakes belonging to the same stack are ordered on the same column over a length $L_{a}$ $\left(S \sim B_{\phi} \ln \left(\beta B_{\phi} / B\right)\right)$ and that where vortex lines are dissociated into single pancakes occupying different columnar defects $\left(S \sim \ln \left(\beta B_{\phi} / B\right)\right)$. The points for $B_{\phi}=0.2$ and 0.5 $\mathrm{T}$ are in fact upper limits for $T S$, obtained from the error bar in the magnetization measurements.

established the value of $\lambda(0)$ with confidence, we use it to evaluate the theoretical logarithmic slope $\partial M_{\text {rev }}^{0} / \partial \ln B=\varepsilon_{0}(T) / 2 \Phi_{0}$ (drawn in Fig. 2). The close coincidence with the data points for $B \gg B_{\phi}$ indicates that in this field regime the entropy contribution is smaller than the experimental error bar. The nearequality of $M_{r e v}\left(B \gg B_{\phi}\right)$ with the magnetization of unirradiated crystals indicates that in either case the entropy in the London regime is very modest indeed, and that vortex positional fluctuations account for only a small correction to the total free energy.

The situation is very different for the regime $B \ll B_{\phi}$, in which all vortices are localized on a columnar defect, and where the experimental $\partial M_{\text {rev }} / \partial \ln B$ lies clearly below $\varepsilon_{0}(T) / 2 \Phi_{0}$. Although the difference is smaller than the value $k_{B} T / \Phi_{0} s$ expected from theory [24], the fact that it exists indicates that entropy is important in the low field regime. This is further borne out by Fig. 3, which shows the reversible magnetization of a series of lightly overdoped crystals with different numbers of sites available to vortices, i.e. with different defect density $n_{d}$ or matching field $B_{\phi}$. It appears that $M_{\text {rev }}$ strongly depends on the density of pinning sites at low fields $\left(B \ll B_{\phi}\right)$, but not at high fields. A strong $B_{\phi}$-dependence of the low-field magnetization cannot be explained in terms of pinning only, but is consistent 
with a substantial entropy associated with the possibility for pancake vortices to occupy different columnar defect sites.

The matching-field dependence of the entropy may be extracted as follows. First, subtract the magnetization of the unirradiated crystal from the experimental curves. In the limit $B \ll B_{\phi}$ this yields a quantity, which, when multiplied by $\Phi_{0} s$, is exactly equal to the sum of the pinning energy and the entropy per pancake vortex. Next, we observe that for relatively small matching fields, $B_{\phi} \leq 0.5 \mathrm{~T}, \partial M_{r e v} / \partial \ln B$ in the lowfield and high-field limits are nearly equal. This implies that for these matching fields, the entropy is small with respect to the pinning energy over the entire investigated field range. We can then confidently determine the pinning energy per pancake $U_{0} s$, either by a fit to a suitable expression, e.g. that given in Ref. [20],

$$
M_{\text {rev }}=M_{r e v}^{0}+\frac{U_{0}}{\Phi_{0}}\left[1-\left(1+\frac{B_{\phi}}{B}\right) \exp \left(-\frac{B_{\phi}}{B}\right)\right]
$$

or by simple identification of the low-field plateau in the $B_{\phi}=0.5 \mathrm{~T}$ data of Fig. $3(\mathrm{~b})$ with $U_{0} s$. The entropy contribution to the free energy follows as the difference between the data and the pinning energy, i.e. $T S=$ $\Phi_{0} s\left(M_{r e v}-M_{r e v}^{0}\right)-U_{0} s$. It is depicted in Fig. 4. We find that the entropy is small at modest $B_{\phi}$, then rapidly rises between $B_{\phi}=0.5 \mathrm{~T}$ and $1 \mathrm{~T}$, after which there is a further increase proportional to $\ln B_{\phi}$. Fig. 3 shows that in the latter regime $T S$ is proportional to $\ln B$.

We interpret this behaviour as follows. At very small matching fields $B_{\phi} \ll B_{c r}$, the (attractive) electromagnetic and Josephson interaction between pancakes in adjacent layers prohibits vortex wandering between columnar defect sites; in this regime entropy can only be gained at the expense of pinning energy by vortex wandering into the intercolumn space $[27,28]$. Evidence for this entropic reduction of the pinning energy for very small matching fields was obtained in Refs. [7,8]. As the density of sites is increased (to above $6 B_{c r}$, see below), vortex lines can gain free energy by spreading to neighbouring columns. The entropy gain in this regime occurs at the expense of elastic (shear and tilt) energy only, and can be estimated as $T S=k_{B} T \ln W=k_{B} T \ln N_{c} L_{s} / L_{a}$, where $L_{s}$ is the thickness of the sample in the field direction, La is the distance over which pancakes belonging to the same vortex line remain ordered on the same columnar track, and $N c \equiv \beta B_{\phi} / B$ is the number of columns available to any one vortex. One then has

$$
M_{t h}=\frac{k_{B} T}{\Phi_{0} s} \frac{s}{L_{a}} \ln \left(\frac{\beta B_{\phi}}{B}\right)
$$

From the extrapolation of the low field data in Fig.3 (b) to $U_{0} s$ we obtain $\beta \sim 0.5$. Balancing the gain in thermal energy and the loss in tilt deformation energy gives $L_{a}=$ $\varepsilon_{0} /\left(\gamma n_{d} k_{B} T\right)$ and

$$
M_{t h}=\frac{k_{B} T}{\Phi_{0} s} \frac{k_{B} T}{\varepsilon_{0} s} \frac{B_{\phi}}{B_{c r}} \ln \left(\frac{\beta B_{\phi}}{B}\right)
$$

in agreement with the rapid rise between $B_{\phi}=0.5 \mathrm{~T}$ and $B_{\phi}=1 \mathrm{~T}$ (see Fig. 4). The typical stacklength can be obtained as the ratio between the experimental entropy values and $\left(k_{B} T / \Phi_{0} s\right)$. As the defect density increases, $L_{a} \sim s\left(\varepsilon_{0} s / k_{B} T\right)\left(B_{c r} / B_{\phi}\right)$ decreases, until at $B_{\phi} \gg B_{c r}\left(\varepsilon_{0} s / k_{B} T\right)$ it becomes equal to the layer spacing s. Experimentally this crossover occurs at a temperature dependent matching field. For $T=74 \mathrm{~K}$, it is at $B_{\phi} \sim 1 \mathrm{~T}$. When the alignment length has decreased to a single pancake vortex, further entropy gain can only be obtained through the supplementary defect sites made available to the pancakes at still higher $B_{\phi}$; then, following Ref. [24],

$$
M_{t h}=\frac{k_{B} T}{\Phi_{0} s} \ln \left(\frac{\beta B_{\phi}}{B}\right)
$$

in very good agreement with the experimental data shown in Figs. 3 and 4.

The field dependence of the reversible magnetization is explained as follows. For the lower matching fields $B_{\phi} \leq 0.5 \mathrm{~T}$ the entropy contribution is negligible with respect to the pinning energy, hence the increase of $\left|M_{\text {rev }}\right|$ at $H_{\text {int }}$ (labelled in Fig. 3) must be ascribed to the reduction of the total pinning energy due to intraplane pancake repulsion. This can be accompanied by the appearance of the first interstitial vortices. At higher matching fields, the low-field magnetization is the sum of $M_{r e v}^{0}$, the gain in pinning energy per pancake $U_{0} s$, and the entropy gain (4) arising from the fact that each pancake can occupy different columns. As the field is increased, less sites are available per pancake, and the entropy contribution decreases logarithmically, until, at $H_{\text {int }}$, intraplane repulsion forces pancakes belonging to the same stack to lign up on the same column. This causes the rapid drop of $M_{t h}$, which is now described by Eq. (2), and the increase of $\left|M_{r e v}\right|$. As the matching field is approached, the increase of $\left|M_{r e v}\right|$ is further enhanced by the decrease in pinning energy and the appearance of interstitial vortices. 


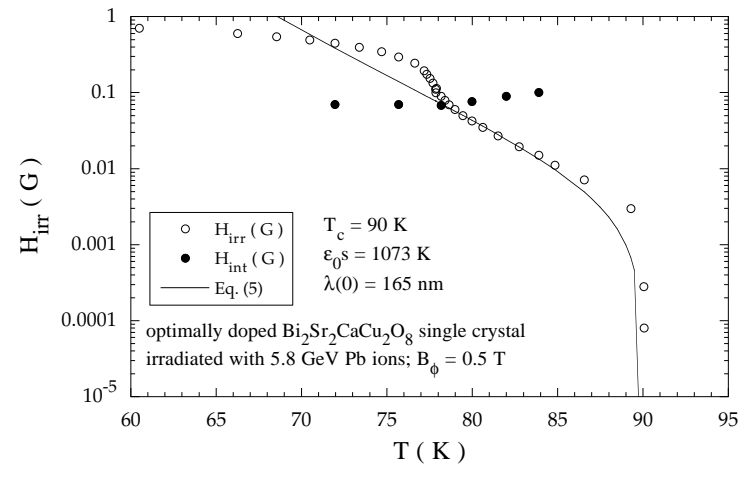

Figure 5: Irreversibility line for an optimally doped $\mathrm{Bi}_{2} \mathrm{Sr}_{2} \mathrm{CaCu}_{2} \mathrm{O}_{8}$ single crystal (University of Amsterdam) irradiated with matching field $B_{\phi}=0.5 \mathrm{~T}$. The IRL is determined as the temperature at which a third harmonic in the AC shielding current can be first observed upon cooling. The drawn line represents a fit to Eq. (5) with the indicated parameter values.

\subsection{Relation with the phase diagram}

In the following we dicuss the consequences of the pancake vortex arrangement for the mixed state phase diagram and vortex dynamics. Fig. 5 shows the IRL determined on an optimally doped $\mathrm{Bi}_{2} \mathrm{Sr}_{2} \mathrm{CaCu}_{2} \mathrm{O}_{8+\delta}$ crystal with $B_{\phi}=0.5 \mathrm{~T}$, together with the $H_{\text {int }}$-line above which intraplane vortex repulsion limits the free energy gain due to columnar defect pinning. The $H_{\text {int }}{ }^{-}$ line depicted in Fig. 5 agrees very well with the "recoupling transition" line found in the JPR experiments of Refs. [13,14].

The irreversibility line has three well-defined parts. At low fields, $H_{i r r}(T)$ increases exponentially

as temperature is lowered. When the interaction (or "recoupling") field $H_{\text {int }}$ is reached, the exponential increase stops and $H_{i r r}$ rises sharply. Thus, at the precise field where intraplane vortex repulsion starts to determine the vortex arrangement in the columnar defect potential, vortex localization is spectacularly improved and vortex dynamics slowed down. Above Hint, every vortex line is localized not only by the defect potential but also by the potential created by the neighbouring vortices. As field is increased further, $H_{\text {irr }}$ eventually starts to decrease roughly linearly with temperature, presumably because the proliferation of weaklylocalized interstitial vortices determines flux dynamics.

The behaviour of the IRL for different matching

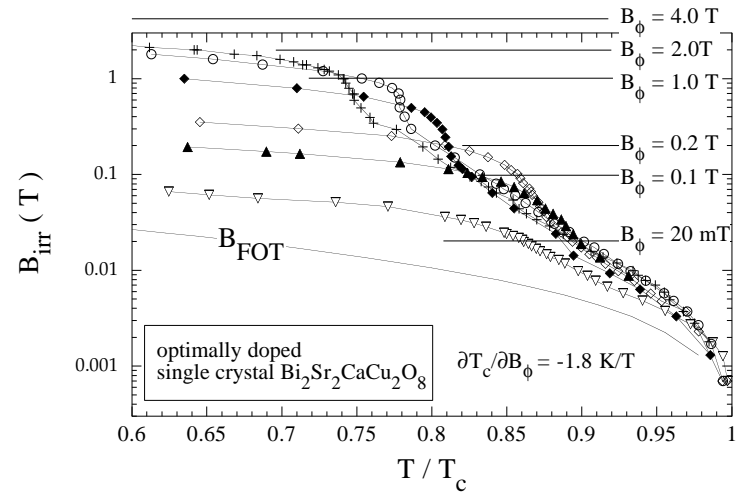

Figure 6: Irreversibility lines for optimally doped Bi2Sr2CaCu2O8 single crystals (University of Tokyo) with different matching fields $0<B_{\phi}<$ T. The line labelled $B_{F O T}$ indicates the first order transition field in the pristine crystal. Other lines are guides to the eye.

fields is shown in Fig. 6. In the low-field, "exponential" regime, $H_{i r r}$ increases monotonically with ion dose up to $B_{\phi} \sim 30 \mathrm{mT}$, after which it saturates to a dose-independent exponential dependence on temperature. Such a saturation of the irreversibility field is reminiscent of experiments on heavy-ion irradiated $\mathrm{YBa}_{2} \mathrm{Cu}_{3} \mathrm{O}_{7-\delta}$, where it signals the stability limit of the entangled vortex liquid with respect to the introduction of correlated disorder. In the low-matching field regime below the saturation (i.e. $B_{\phi}<30 \mathrm{mT}$ ), the exponential temperature dependence of $H_{i r r}$ can be observed up to $H_{i r r} B_{\phi}$, after which it crosses over to a linear dependence $H_{i r r} \sim(1-T / T c)$. For $B_{\phi}>30 \mathrm{mT}$, the abovementioned break at $H_{\text {int }} \sim B_{\phi} / 6$ appears. It is found for the whole investigated range $0.1 \mathrm{~T}<B_{\phi}<4$ $\mathrm{T}$, always near the same fraction $B_{\phi} / 6$. At fields above $B_{\phi} / 6, H_{i r r}$ decreases with increasing matching field. This is

again the result of the fact that an increase of the number of available columnar defect sites promotes vortex wandering and eventually, diffusion.

\subsection{Vortex delocalization in the low-field limit}

The low-field regime $H<H_{\text {int }}$ in which the irreversibility line saturates to a $B_{\phi}$-independent exponential temperature dependence is that in which interactions between different vortex lines are irrelevant, and, there- 
fore, where every pancake vortex can find a suitable columnar defect site. Moreover, since for $B_{\phi} \leq 0.5 \mathrm{~T}$, pancakes belonging to the same vortex line are wellaligned even in the vortex liquid $\left(L_{a} \sim 10 \mathrm{~s}\right.$ at 74 $\mathrm{K})$, the coincidence of the exponential part of the irreversibility lines for $0.1 \mathrm{~T}<B_{\phi}<4 \mathrm{~T}$ implies that near $H_{i r r}(T)$ pancakes belonging to the same stack are aligned on the same ion track irrespective of the matching field. Vortex motion and delocalization can then be modelled in terms of a "discrete superconductor" in which the only sites allowed to the vortices are defect sites. The absence of strong positional fluctuations inferred in Sections 3.1 and 3.2 implies that the main thermal fluctuations will be occasional single-pancake jumps to neighbouring columns. These can be seen as bound vacancy-interstitial pairs (or "quartets" [30]), with separation $R$ and energy $\varepsilon_{q}=\varepsilon_{0} s(R / \gamma s)^{2}$, present in the vortex system of the discrete superconductor. The onset of vortex diffusion at the IRL corresponds to the defect pair unbinding; hence, $k_{B} T_{i r r} \sim \varepsilon_{q}\left(R_{l}\right)$ where $R_{l} \sim\left(\Phi_{0} / B\right)^{1 / 2} \exp \left(-\varepsilon_{0} s / 2 k_{B} T\right)$ is the typical distance between free dislocations (free pancakes) in the vortex liquid. Gathering terms, one has $k_{B} T_{i r r}=$ $\varepsilon_{0} s\left(\Phi_{0} / \gamma^{2} s^{2}\right) \exp \left(\varepsilon_{0} s / k_{B} T\right)$ or

$$
B_{i r r}=B_{c r} \frac{\varepsilon_{0} s}{k_{B} T} \exp \left(\frac{\varepsilon_{0} s}{k_{B} T}\right)\left(B_{c r} \ll B<H_{i n t}\right)
$$

which satisfactorily describes the exponential part of the IRL, with parameters $\lambda(0)=165 \mathrm{~nm}$ and $B_{c r}=$ $6.5 \mathrm{mT}$ (i.e. $\gamma \approx 350$ ) (see Fig. 5). The upper limit of applicability of Eq. (5), as this emerges from Section 3.3 (i.e. $B<H_{\text {int }}=B_{\phi} / 6$ ) also gives the lowest $B_{\phi}$ -value for which Eq. (5) applies: $B_{\phi}=6 B_{c r} \sim 40 \mathrm{mT}$, in very good agreement with the matching field value at which the IRL saturates. We conclude that the value $B_{\phi}=6 B_{c r}$ delimits the low defect-density regime, in which thermal vortex excursions into the intercolumn space are allowed, from the high-defect density regime where the "discrete superconductor"-model of strongly localized vortices is applicable.

\section{Conclusions}

Reversible magnetization measurements on $\mathrm{Bi}_{2} \mathrm{Sr}_{2} \mathrm{CaCu}_{2} \mathrm{O}_{8+\delta}$ single crystals containing amorphous columnar defects introduced by heavy-ion irradiation are used to determine the magnitude of the pinning energy and entropy contributions to the mixed state free energy. Whereas the pinning energy contribution can always be identified, the importance of entropy depends strongly on the defect density and the magnetic field. For low defect densities and unirradiated crystals, the entropy contribution to the free energy in the London regime (i.e. the entropy gain associated with vortex positional fluctuations) is found to be very minor with respect to the vortex core, electromagnetic, and pinning energy contributions. It therefore does not determine the main features of the phase diagram. At higher matching fields (typically above $1 \mathrm{~T}$ ) and low magnetic fields $B<H_{\text {int }} B_{\phi} / 6$, the entropy associated with the possibility of pancakes belonging to the same vortex line to occupy different defect sites becomes important. This configurational entropy greatly exceeds the fluctuation entropy in unirradiated samples; it can attain values that are more than twice the pinning energy, for $B_{\phi} \sim 4 \mathrm{~T}$. When the magnetic field is increased above $H_{\text {int }}$, intraplane pancake vortex repulsion causes the total pinning energy and the configurational entropy to diminish. Nevertheless, vortices become more strongly localized, since they are now retained not only by the columnar defect potential, but also by that of the neighbouring vortices. This effect is apparent as a steep increase in the IRL, and as the recently found "recoupling" transition in JPR experiments $[13,14]$.

\section{References}

[1] J.R. Thompson, Y.R. Sun, H.R. Kerchner, D.K. Christen, B.C. Sales, B.C. Chakoumakous, A.D. Marwick, L. Civale, and J.O. Thompson, Appl. Phys. Lett. 60, 2306 (1992).

[2] W. Gerhäuser, G. Ries, H.W. Neumüller, W. Schmidt, O. Eibl, G. Saemann-Ischenko, and S. Klaumünzer, Phys. Rev. Lett. 68, 879 (1992).

[3] M. Koncykowski, Y. Yeshurun, L. Klein, E.R. Yacoby, N. Chikumoto, V.M. Vinokur, and M.V. Feigel'man, J. Alloys Comp. 195, 407 (1993).

[4] M. Konczykowski, N. Chikumoto, V.M. Vinokur, and M.V. Feigel'man, Phys. Rev. B 51, 3957 (1995).

[5] M. Konczykowski, Physica C 235-240, 197 (1994); M. Konczykowski, N. Chikumoto, V. Vinokur, M. Feigel'man, Physica C 235-240, 2845 (1994).

[6] L. Klein, E.R. Yacoby, Y. Yeshurun, M. Konczykowski, and K. Kishio, Phys. Rev B 48, 4403 (1994).

[7] C.J. van der Beek, M. Konczykowski, V.M. Vinokur, T.W. Li, P.H. Kes, and G.W. Crabtree, Phys. Rev. B 51, (1995). 
[8] C.J. van der Beek, M. Konczykowski, V.M. Vinokur, T.W. Li, P.H. Kes, and G.W. Crabtree, Phys. Rev. Lett. 74, 1214 (1995).

[9] R. Doyle, W.S. Seow, Y. Yan, A.M. Campbell, T. Mochiku, K. Kadowaki, and G. Wirth, Phys Rev. Lett., Phys. Rev. Lett. 77, 1155 (1995).

[10] N. Morozov, M.P. Maley, L.N. Bulaevskii, and J. Sarrao, Phys. Rev. B 57, R8146 (1998).

[11] N. Morozov, M.P. Maley, L.N. Bulaevskii, V. Thorsmølle, A.E. Koshelev, A. Petrean, and W.K. Kwok, Phys. Rev. Lett. 82, 1008 (1999).

[12] T. Hanaguri, Y. Tsuchiya, S. Sakamoto, A. Maeda, and D.G. Steel, Phys. Rev. Lett. 78, 3177 (1997).

[13] M. Sato, T. Shibauchi, S. Ooi, T. Tamegai, and M. Konczykowski, Phys. Rev. Lett. 79, 3759 (1997).

[14] M. Kosugi, Y. Matsuda, M.B. Gaifullin, L.N. Bulaevskii, N. Chikumoto, M. Konczykowski, J. Shimoyama, K. Kishio, K. Hirata, and K. Kumagai, Phys. Rev. Lett. 79, 3763 (1997).

[15] L.N. Bulaevskii, M.P. Maley, and V.M. Vinokur, Phys. Rev. B 57, R5626 (1998).

[16] N. Chikumoto, M. Kosugi, Y. Matsuda, M. Konczykowski, and K. Kishio, Phys. Rev. B 57, 14507 (1998).

[17] U.C. Täuber, H.J. Dai, D.R. Nelson, and C.M. Lieber, Phys. Rev. Lett. 74, 5132 (1995); U.C. Täuber and D.R. Nelson, Phys. Rev. B 52, 16106 (1995).

[18] C. Wengel and U.C. Täuber, Phys. Rev. Lett. 78, 4845 (1997); C. Wengel and U.C. Täuber, Phys. Rev. B 58, 6565 (1998).

[19] J. Gilchrist and M. Konczykowski, Physica (Amsterdam) C 212, 43 (1993).

[20] A. Wahl, V. Hardy, J. Provost, C. Simon, and A. Buzdin, Physica C 250, 163 (1995).

[21] C.J. van der Beek, M. Konczykowski, T.W. Li, P.H. Kes, and W. Benoit, Phys. Rev. B. 54, R792 (1996).

[22] R.J. Drost, C.J. van der Beek, H.W. Zandbergen, M. Konczykowski, A.A. Menovsky, and P.H. Kes, Phys. Rev. B 60, (1998).

[23] L.N. Bulaevskii, M. Ledvij, and V.G. Kogan, Phys. Rev. Lett. 68, 3773 (1992).

[24] L.N. Bulaevskii, V.M. Vinokur, and M.P. Maley, Phys. Rev. Lett. 77, 936 (1996).

[25] Note that the ratio between these two terms is just 2Gi1/2, where Gi is the two-dimensional Ginzburg number. In optimally doped $\mathrm{Bi} 2 \mathrm{Sr} 2 \mathrm{CaCu} 2 \mathrm{O} 8+\mathrm{d}, \mathrm{Gi}=$ 0.01 .

[26] This $\lambda(0)$ value is approximately $4 \%$ larger than that in unirradiated crystals. Namely, the large density of columnar defects $n_{d} \gg \lambda^{-2}$ entails a reduction of the superfluid density and a renormalization of the vortex currents, which can be expressed by the substitution $\lambda^{-2} \rightarrow \lambda^{-2}\left(1-2 \pi c_{0}^{2} n_{d}\right)$
[27] D.R. Nelson and V.M. Vinokur, Phys. Rev. Lett. 68, 2398 (1992); Phys. Rev. B 48, 13060 (1993).

[28] A.E. Koshelev, P. Le Doussal, and V. M. Vinokur, Phys. Rev. B 53, R8855 (1996); and unpublished.

[29] A.V. Samoilov, M.V. Feigel'man, M. Konczykowski, and F. Holtzberg, Phys. Rev. Lett. 76, 2798 (1996).

[30] M.V. Feigel'man, V.B. Geshkenbien, and A.I. Larkin, Physica (Amsterdam) C 167, 117 (1990) 